\title{
S100A13 is a new angiogenic marker in human melanoma
}

\author{
Daniela Massi ${ }^{1,6}$, Matteo Landriscina ${ }^{2,6}$, Annamaria Piscazzi ${ }^{2}$, Elena Cosci ${ }^{3}$, Alek Kirov, \\ Milena Paglierani ${ }^{1}$, Claudia Di Serio ${ }^{5}$, Vasileios Mourmouras ${ }^{3}$, Stefano Fumagalli ${ }^{5}$, \\ Maurizio Biagioli ${ }^{3}$, Igor Prudovsky ${ }^{4}$, Clelia Miracco ${ }^{3}$, Marco Santucci $^{1}$, \\ Niccolò Marchionni ${ }^{5}$ and Francesca Tarantini ${ }^{5}$ \\ ${ }^{1}$ Division of Pathological Anatomy, Department of Critical Care Medicine and Surgery, University of Florence, \\ Florence, Italy; ${ }^{2}$ Clinical Oncology Unit, Department of Medical Sciences, University of Foggia, Foggia, Italy; \\ ${ }^{3}$ Department of Human Pathology and Oncology, Section of Pathological Anatomy, University of Siena, \\ Siena, Italy; ${ }^{4}$ Maine Medical Center Research Institute, Scarborough, ME, USA and ${ }^{5}$ Geriatric Medicine Unit, \\ Department of Critical Care Medicine and Surgery, University of Florence, Florence, Italy
}

\begin{abstract}
Angiogenesis is critical in melanoma progression and metastasis and relies on the synthesis and release of proangiogenic molecules such as vascular endothelial growth factor (VEGF)-A and fibroblast growth factors (FGFs). S100A13 is a small calcium-binding protein that facilitates the release of FGF-1, the prototype of the FGF family. S100A13 is upregulated in astrocytic gliomas, in which it correlates with VEGF-A expression, microvessel density and tumor grading, and promotes a more aggressive, invasive phenotype in lung cancer-derived cell lines. To investigate the involvement of S100A13 in human cutaneous melanoma, we analyzed a series of 87 cutaneous melanocytic lesions: 14 common acquired melanocytic nevi, 14 atypical, so-called 'dysplastic' nevi, 45 melanomas (17 radial growth phase and 28 vertical growth phase) and 14 melanoma metastases. Main clinical and pathological features, including histotype, Breslow thickness, Clark's level and outcome were recorded. Microvessel density was determined with CD105/endoglin staining. Semiquantitative determination of S100A13, FGF-1 and VEGF-A protein expression was obtained by immunostaining. Quantification of S100A13 mRNA was achieved by real-time PCR. We found that S100A13 was expressed in melanocytic lesions; compared with benign nevi, S100A13 protein expression was significantly upregulated in melanomas $(P=0.024)$, in which it correlated positively with the intensity of VEGF-A staining $(P=0.041)$ and microvessel density $(P=0.007)$. The level of expression of S100A13 mRNA also significantly increased with progression of disease, from radial growth phase $(0.7 \pm 0.7)$ to vertical growth phase (3.6 \pm 3.1) to metastases $(7.0 \pm 7.0)(P<0.001)$. Furthermore, S100A13 mRNA correlated positively with VEGF-A $(P=0.023)$, TNM stage $(P=0.05)$, risk of relapse $(P=0.014)$ and status at follow-up $(P=0.024)$. In conclusion, S100A13 is expressed in melanocytic lesions when the angiogenic switch occurs and it may cooperate with VEGF-A in supporting the formation of new blood vessels, favoring the shift from radial to vertical tumor growth. Therefore, S100A13 may represent a new angiogenic and prognostic marker in melanoma.
\end{abstract}

Modern Pathology (2010) 23, 804-813; doi:10.1038/modpathol.2010.54; published online 5 March 2010

Keywords: melanoma; tumor angiogenesis; S100A13; FGF-1; immunohistochemistry; real-time PCR

Tumor angiogenesis, leading to the development of new blood vessels, represents a crucial step to

Correspondence: Associate Professor F Tarantini, MD, PhD, Geriatric Medicine Unit, Department of Critical Care Medicine and Surgery, School of Medicine, University of Florence, Viale Pieraccini n. 6, Florence 50139, Italy.

E-mail: taranf@unifi.it

${ }^{6}$ These authors contributed equally to the work.

Received 16 December 2009; revised 16 January 2010; accepted 19 January 2010; published online 5 March 2010 ensure a supply of nutrients and oxygen to the growing mass and to provide a route for metastatization. Angiogenesis depends on the ability of cancer and stromal cells to switch on the synthesis and release of proangiogenic molecules. ${ }^{1}$ Tumors are able to produce multiple angiogenic polypeptides; however, vascular endothelial growth factor (VEGF)-A and the prototypes of the fibroblast growth factor (FGF) family, FGF-1 and FGF-2, are the most widely expressed in cancer tissues. $^{2}$ 
Angiogenesis is a distinct feature of cutaneous melanomas, and it associates with worst outcome. ${ }^{3}$ Although the most critical step in metastases remains the spreading of melanoma cells to the lymphatic vessels surrounding the tumor, the progression of melanoma lesions from a radial growth phase to a vertical growth phase requires high angiogenic activity. ${ }^{4}$ To accomplish this task, transformed melanocytes produce and release large amounts of FGF-1, FGF-2 and VEGF-A. ${ }^{5}$

S100A13 is a small calcium $\left(\mathrm{Ca}^{2+}\right)$-binding protein belonging to the S100 family. ${ }^{6}$ The family members are multifunctional proteins, involved in the regulation of several cellular processes including contraction and motility, cell growth and differentiation, cell cycle progression, organization of membrane structure, control of cytoskeleton assembly, protection from oxidative damage and protein secretion. $^{7}$

Several members of the S100 family, including S100A4, S100A6, S100A7 and S100A10, are overexpressed in cancer cells, compared with normal cells, and foster more aggressive phenotypes. S100A13 is upregulated in human astrocytic gliomas, in which it correlates with VEGF-A expression, microvessel density and tumor grading, ${ }^{8}$ and it is also associated with a more aggressive, invasive phenotype in lung cancer-derived cell lines. ${ }^{9}$

S100A13 is involved in the release of FGF-1 which is devoid of a classical signal peptide sequence to direct secretion through the endoplasmic reticulum (ER)-Golgi apparatus and, therefore, is exported through an 'unconventional' secretory pathway: FGF-1 exits the cell as part of a copperdependent multiprotein complex that includes S100A13, the extravesicular portion of synaptotagmin-1 and sphingosine kinase $1 .^{10} \mathrm{~A}$ dominant negative S100A13 construct is able to block FGF-1 secretion from NIH 3T3 cells, suggesting that the presence and function of S100A13 is necessary for the release of this proangiogenic polypeptide. ${ }^{10-11}$

Melanomas express S100A2, S100A4, S100A6, as well as $\mathrm{S100B}$ that still represents the most sensitive marker for melanocytic lesions. ${ }^{12}$ S100B and S100A6 correlate with tumor burden and prognosis, whereas S100A2 displays an inverse correlation with malignancy. No relationship with prognosis was demonstrated for S100A4 gene expression. ${ }^{13-16}$

This study was designed to determine whether S100A13 is expressed in melanocytic lesions and has a role in human melanoma progression.

\section{Materials and methods}

\section{Specimen Selection}

Tissues were obtained from the archive of the Department of Human Pathology and Oncology, University of Siena. The study series included 87 cutaneous melanocytic lesions: 28 melanocytic nevi and 59 melanomas. Immunohistochemical studies of CD105 and VEGF-A expression in a part of this series have been reported in a earlier publication. ${ }^{17}$ Melanocytic nevi were distributed as follows: 14 benign nevi (4 common acquired nevi; 7 Spitz/Reed nevi; and 3 blue nevi) and 14 so-called atypical, 'dysplastic' nevi. Melanomas were distributed as follows: 17 primary lesions in radial growth phase and 28 in vertical growth phase, and 14 metastases (12 cutaneous metastases and 2 from central nervous system metastatic deposits). Age at diagnosis, sex, anatomic location of melanocytic lesion, TNM stage (AJCC 2002, 6th edition) and outcome were recorded for each patient. Disease relapses included nodal recurrences (recurrences in the draining nodal basin from the primary lesion), regional recurrences (which included local and in-transit lesions) and systemic disease (lesions in all other locations). Follow-up ranged from 12 to 90 months (mean 39 months; median 39 months). Mean and median follow-up of patients who did not develop disease relapse were 41 and 39 months, respectively. Histopathological slides were examined for histotype, growth phase (radial or vertical), Breslow thickness, Clark's level, presence of ulceration and mitotic activity. Table 1 summarizes the main clinical and pathological features of patients with melanoma. The protocol was approved by the institutional review board for use of human tissues.

\section{Immunohistochemistry}

Immunohistochemistry was performed on sections of $4 \mu \mathrm{m}$ thickness prepared from formalin-fixed and paraffin-embedded tissues, using the streptavidinbiotin peroxidase complex method. Sections were deparaffinized in Bio-Clear (Bio-Optica, Milano, Italy) and hydrated with graded ethanol and distilled water. Antigen retrieval was routinely performed by immersing the slides in thermostatic bath containing preheated $10 \mathrm{mM} / \mathrm{l}$ citrate buffer ( $\mathrm{pH}$ 6.0) for $30 \mathrm{~min}$ at $98^{\circ} \mathrm{C}$ and cooling down at room temperature for $20 \mathrm{~min}$. To block endogenous peroxidase activity, the slides were treated with $3.0 \%$ hydrogen peroxidase in distilled water for $10 \mathrm{~min}$, followed by three washes in Tris/HCl buffer. For detection of CD105/endoglin, after pretreatment with protease for $10 \mathrm{~min}$, the anti-CD105 monoclonal antibody (clone SN6H, Dako Denmark A/S, Glostrup, DK) (1:50 dilution) was applied overnight at $4^{\circ} \mathrm{C}$. For detection of VEGF-A, after pretreatment in Tris/EDTA/citrate buffer, $\mathrm{pH}$ 8.0, for $30 \mathrm{~min}$, the anti-human VEGF-A monoclonal antibody (clone VG1, identifying the VEGF-A isoforms VEGF121, VEGF165 and VEGF189; DAKO 1:100 dilution) was applied overnight at $4^{\circ} \mathrm{C}$. For signal amplification, we used the UltraVision LP Large Volume Detection System AP Polymer (Ready-To-Use) (Bio-Optica, Milan, Italy), with New Fuchsin (Bio-Optica) as chromogen, developing a cytoplasmic red stain in positive cells. For S100A13 immunohistochemical 
Table 1 Clinical and histopathological features of patients with melanoma (primary and metastatic)

\begin{tabular}{|c|c|c|c|}
\hline & $R G P(\mathrm{n}=17)$ & $V G P(\mathrm{n}=28)$ & $M M(\mathrm{n}=14)$ \\
\hline Male/age & $5 / 59$ & $18 / 61$ & $8 / 71$ \\
\hline \multicolumn{4}{|l|}{ Site } \\
\hline Trunk & 8 & 14 & 4 \\
\hline Leg & 6 & 4 & 3 \\
\hline Head/neck & 1 & 3 & 4 \\
\hline Arm & 2 & 5 & 3 \\
\hline Acral & 0 & 2 & 0 \\
\hline \multicolumn{4}{|l|}{ Histotype } \\
\hline SSM & 17 & 19 & \\
\hline NM & 0 & 7 & \\
\hline ALM & 0 & 2 & \\
\hline \multicolumn{4}{|l|}{ Level } \\
\hline I & 10 & 0 & \\
\hline II & 7 & 0 & \\
\hline III & 0 & 3 & \\
\hline IV & 0 & 23 & \\
\hline $\mathrm{V}$ & 0 & 2 & \\
\hline Thickness (mm) & & 0.14 & 3.41 \\
\hline Mitosis $\left(\mathrm{mm}^{2}\right)$ & & 0.34 & 8.8 \\
\hline Ulceration (n) & & 0 & 16 \\
\hline \multicolumn{4}{|l|}{ TNM Stage } \\
\hline 0 & 8 & 0 & \\
\hline I & 8 & 10 & \\
\hline II & 1 & 12 & \\
\hline III & 0 & 3 & \\
\hline IV & 0 & 3 & 14 \\
\hline Follow-up (months) & 42.6 & 41 & 33.6 \\
\hline Relapse at follow-up & 0 & 8 & 14 \\
\hline \multicolumn{4}{|l|}{ Status } \\
\hline Aw/oD & 17 & 18 & 0 \\
\hline AwD & 0 & 4 & 2 \\
\hline DfD & 0 & 6 & 12 \\
\hline
\end{tabular}

RGP, radial growth phase; VGP, vertical growth phase; MM, melanoma metastases; NM, nodular melanoma; SSM, superficial spreading melanoma ALM, acral lentiginous melanoma; Aw/oD, alive without disease; AwD, alive with disease; DfD, dead for disease.

analysis, sections were incubated with rabbit antihuman S100A13 polyclonal antibody ${ }^{11}$ at 1:50 dilution in Antibody diluent (Ventana Medical Systems, Tucson, AZ, USA) for $30 \mathrm{~min}$ at room temperature. Staining was achieved using the EnVision/HRP Plus mouse kit (Dako, Carpinteria, CA, USA) with aminoethylcarbazol (UltraVision, Fremont, CA, USA) as chromogen. All sections were counterstained with Mayer's hematoxylin.

Negative controls were performed by substituting the primary antibody with a non-immune serum at the same concentration. Control sections were treated at the same time as sample sections.

\section{Assessment of VEGF-A and S100A13 Immunostaining}

VEGF-A immunostaining was evaluated by two independent observers all throughout tumor areas, according to a semiquantitative score based on both the stain intensity (1: weak; 2: moderate; 3: strong), and the percentage of positive melanocytes $(\leqslant 25 \%$ : $1 ; 26-50 \%: 2 ; 51-75 \%: 3 ;>75 \%$ : 4). A final value of 0 was assigned to negative cases, and a value of 1,2 and 3 to cases scored $2-3,4-5$ and $6-7$, respectively (low, moderate and strong VEGF-A expression). ${ }^{17}$ S100A13 immunostaining was classified according to a semiquantitative score, ranging from 0 to 4 , based on the percentage of positive melanocytes ( $\leqslant 25 \%: 1 ; 26-50 \%$ : $2 ; 51-75 \%: 3 ;>75 \%: 4$ ).

\section{Assessment of Microvessel Density}

To assess mean microvessel density, CD105-stained sections were scanned at low magnifications $(\times 25$ and $\times 100)$ to identify the most vascularized areas of the tumor (hot spots). In thin lesions (junctional melanocytic nevi, in situ and thin melanomas), the evaluation was limited to one high power field (HPF, $\times 40$ objective and 10 eyepiece) in the tumorstroma boundary; in thick lesions (compound nevi; VGP melanomas) and metastases, vessel counts were performed in the central/vertical growth phase area and at the peripheral tumor-stroma boundary, within one HPF in the tumor invasive front. Vessels more than one-half HPF away from the above defined areas, as well as vessels inside necrotic and ulcerated areas, were not counted. From a minimum of 5 to a maximum of 10 randomly chosen fields were evaluated by two independent observers, at $\times 400$ magnification (HPF, $0.16 \mathrm{~mm}^{2}$ / field) and the mean value was calculated for statistical analysis. ${ }^{17}$

\section{Quantitative RT-PCR Analysis}

Total RNA was extracted from tumor-enriched frozen fragments, using SV Total RNA isolation kit (Promega, Milan, Italy). For cDNA synthesis, RNA (500 ng) was reverse transcribed in a final volume of $20 \mu \mathrm{l}$, containing $1 \mu \mathrm{l}$ of dNTPs $10 \mathrm{mM} ; 4 \mu \mathrm{l}$ of $5 \times$ first-strand buffer; $0.6 \mu \mathrm{l}$ (24U) of RNAsis RNase inhibitor (Promega, Florence, Italy); $2 \mu \mathrm{l}$ of $0.1 \mathrm{M}$ DDT; $1 \mu \mathrm{l}$ (200 U) of M-MLV Reverse Transcriptase (Invitrogen, San Diego, CA, USA); $0.5 \mu \mathrm{l}$ (200 ng) random hexamers (Pharmacia, Milan, Italy). Samples were then incubated at $25^{\circ} \mathrm{C}$ for $10 \mathrm{~min}$, and $37^{\circ} \mathrm{C}$ for $50 \mathrm{~min}$. Reverse transcriptase was inactivated by heating at $70^{\circ} \mathrm{C}$ for $15 \mathrm{~min}$. No RT controls were performed by the addition of nuclease-free water. All products were stored at $-20^{\circ} \mathrm{C}$ until use. For quantitative PCR analysis, $1 \mu \mathrm{l}$ of cDNA was amplified using the Platinum SYBR Green qPCR Supermix UDG (Invitrogen) in an iCycler iQ Real Time Detection System (Biorad Laboratories GmbH, Muenchen, Germany). The following primers were used: S100A13 forward 5'-TCCTAATGGCAGCAGA ACCACTGA- $3^{\prime}$, reverse $5^{\prime}$-TTCTTCCTGATTTCCT TGGCCAGC-3', (PCR product $273 \mathrm{bp}$ ); GAPDH, forward 5'-CAAGGCTGAGAACGGGAA-3', reverse 
5'-GCATCGCCCCACTTGATTTT-3' (PCR product $90 \mathrm{bp}$ ). Primers were designed to be intron-spanning. Reaction conditions were $50^{\circ} \mathrm{C}$ for $2 \mathrm{~min}, 95^{\circ} \mathrm{C}$ for $2 \mathrm{~min}$, followed by 45 cycles of $95^{\circ} \mathrm{C}$ for $15 \mathrm{~s}$, $60^{\circ} \mathrm{C}$ for $30 \mathrm{~s}, 72^{\circ} \mathrm{C}$ for $30 \mathrm{~s}$. GAPDH was chosen as an internal control. Absolute quantification was carried out against a standard curve performed by amplification of a plasmid encoding for S100A13 cDNA.

\section{Statistical Analysis}

Statistical analysis was performed with SPSS for Windows (ver. 17) (Chicago, IL, USA). Continuous variables were expressed as mean \pm s.d. or as median value if an abnormal distribution was observed. Categorical variables were expressed as percentages. Student's $t$ test or analysis of variance with Bonferroni's test for post hoc analysis was used to verify the existence of differences in continuous variables between two or more groups of samples, respectively. In all cases we performed the corresponding nonparametric test, namely, Mann-Whitney and Kruskal-Wallis tests. Linear regression analysis was used to study the relation between two continuous variables. For categorical variables $\chi^{2}$-test was used. To further increase the severity of analysis, exact tests were built with Monte Carlo method in all cases. A two-tailed $P$-value $<0.05$ was considered to express statistical significance.

\section{Results}

\section{S100A13 is Expressed in Melanocytic Lesions}

S100A13 protein expression in melanocytic lesions was investigated by immunohistochemistry. In adjacent normal skin, S100A13 was diffusely expressed in basal epidermal keratinocytes, with focal nuclear staining in the suprabasal keratinocytes in the spinous layer, and no significant staining in the granular layer and stratum corneum. A high expression level of S100A13 was consistently observed in the outer and inner root sheath of hair follicles, eccrine sweat glands and sebaceous glands. In blood vessels, a positive immunostaining was detected in vascular smooth muscle cells, while endothelial cells appeared negative. Dermal inflammatory cells were not stained. In common melanocytic nevi, S100A13 staining was generally absent, with only a faint immunoreactivity in a small number of cells. Large epithelioid and spindle cells in common Spitz's nevi were also negative, while 'dysplastic', atypical nevi displayed a variable S100A13 expression: nevi with mild atypia showed weak expression in a minority of melanocytes, whereas 'dysplastic' nevi with moderate-severe atypia displayed diffuse positivity with variable intensity (Figure 1). Cytologically atypical cells displayed a stronger cytoplasmic staining, while nuclear staining was not observed. In melanomas, staining was mostly confined to the cell cytoplasm, with minimal peripheral membrane and rare nuclear pattern. Primary melanomas showed moderate-to-strong S100A13 expression in the majority of tumor cells. In metastatic melanomas, immunoreactivity appeared also increased, although with variable intensity, and it was mostly confined to the cell cytoplasm (Figures 2 and 3).

Statistical analysis demonstrated that S100A13 protein was significantly upregulated in 'dysplastic' nevi and melanomas compared with benign nevi $(P=0.024)$. Because of the semiquantitative nature of S100A13 immunohistochemical analysis, S100A13 gene expression was studied in all samples, in which a frozen fragment was available for RNA extraction: 3 'dysplastic' nevi, 9 radial growth phase, 15 vertical growth phase melanomas and 7 metastases. The main limitation to obtain frozen fragments was represented by the scarce amount of material that is usually recovered during biopsy/ surgical removal of the melanocytic lesion, especially in thin and narrow radial growth phase melanomas, material that was primarily used to obtain the histopathological diagnosis and grading of the tumor.

As shown in Figure 4, there was a significant increase of S100A13 mRNA level from 'dysplastic' nevi and radial growth phase to vertical growth phase melanomas, to metastatic lesions $(P<0.001$, Kruskal-Wallis $H$-test).

\section{Expression of S100A13 Correlates with Invasion and Angiogenesis}

When the expression of S100A13 mRNA was analyzed in relation to the depth of invasion, we found that S100A13 gene expression significantly correlated with Breslow thickness $(0.6 \pm 0.7$ for lesions $<1 \mathrm{~mm}$ vs $3.9 \pm 3.4$ for lesions $>2 \mathrm{~mm}$, $P=0.001$ Mann-Whitney test) and Clark's level ( $\beta=0.21 \pm 0.09, R=0.493, P=0.023$ ).

To explore the angiogenic profile, we performed immunohistochemical analysis of VEGF-A, and assessed microvessel density by counting vessels positive for CD105/endoglin. In the majority of nevi, melanocytes were negative for VEGF-A staining; a cytoplasmic mild expression was observed in a benign common nevus; a mild-to-moderate positivity in 3 Spitz nevi, and a mild expression in a 'dysplastic' nevus. In malignant lesions, VEGF-A expression was more heterogeneous, ranging from negative (14 radial growth phase; 6 vertical growth phase; 2 melanoma metastases), to mild, moderately or strongly positive cases (Figure 5). Furthermore, a heterogeneous expression was usually observed within each lesion. In all lesions, a mild-to-moderate cytoplasmic positivity for VEGF-A was detected in endothelial cells in the majority of vessels, with the strongest positivity at the periphery of malignant lesions; few eccrine gland cells were also stained, 


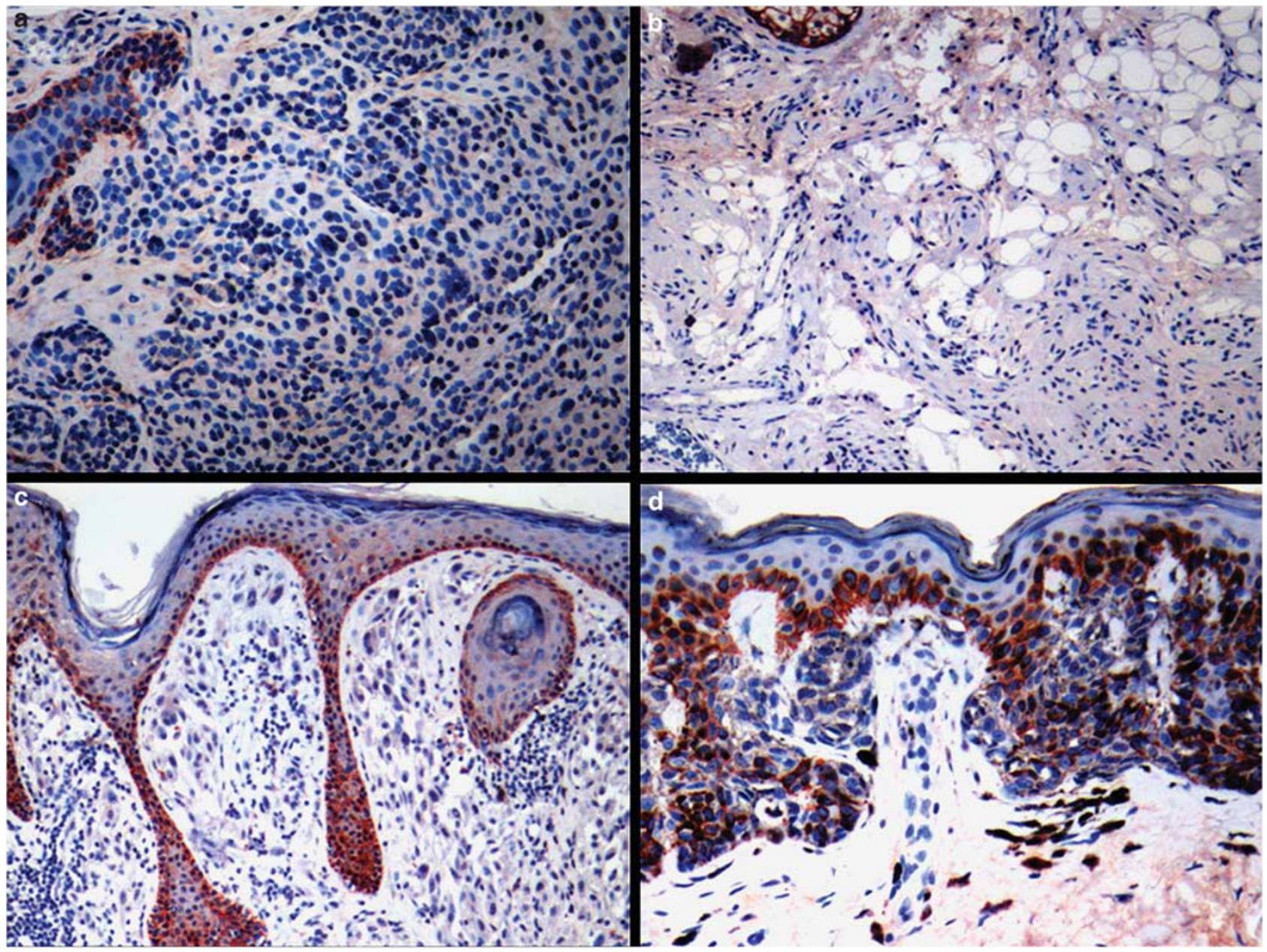

Figure 1 S100A13 staining in melanocytic nevi: (a) absence of S100A13 immunoreactivity in a common acquired nevus. Note that basal epidermal keratinocytes are positive (upper left); (b) cytologically bland melanocytes in a neurotized dermal nevus are S100A13 negative; (c) absence of immunostaining in the spindle and epithelioid melanocytes in a common Spitz's nevus (epidermal basal keratinocytes are positive); (d) 'dysplastic' nevus with mild atypia showing weak S100A13 positivity in a minority of cells.

whereas cells of other adnexa and epidermis, as well as inflammatory, nerve, fat and muscle cells were negative.

Unlike VEGF-A, FGF-1 mRNA and protein were highly expressed in all tissues examined, with no major differences between benign and malignant lesions (data not shown).

As expected, mean intratumoral $(\mathrm{C} / \mathrm{V})$ and peritumoral (P) microvessel density, calculated using CD105 antibodies, were significantly higher in primary and metastatic melanomas, in comparison with melanocytic nevi $(P<0.001$ and $P=0.001$, respectively), with lesions in radial growth displaying a significantly lower number of vessels than vertical growth phase and metastatic melanomas (C/V, radial growth phase $3.2 \pm 0.9$ vs vertical growth phase $7.3 \pm 2.2$ vs metastatic melanomas $7.3 \pm 2.2$, $P<0.001)$. Furthermore, the density of CD105positive blood vessels appeared higher in nevi and melanomas than in neighboring normal human skin (data not shown). The epidermis and adnexal structures were CD105-negative. The intensity of the staining was variable in endothelial cells, although it was constantly strong in melanoma vessels within areas of regression.

VEGF-A staining showed that the expression of the protein was higher in highly vascularized tumors, with a significant correlation with intratumoral microvessel counts (VEGF-A score 0, microvessel density $4.4 \pm 2.0$ vs VEGF-A score 3, microvessel density $7.3 \pm 1.4, P=0.001)$. VEGF-A also correlated with Breslow thickness $(0.7 \pm 1.1$ for lesions $<1 \mathrm{~mm}$ vs $1.4 \pm 1.2$ for lesions $>2 \mathrm{~mm}$, $P=0.05)$ and Clark's level $(\beta=0.7 \pm 0.1, R=0.608$, $P<0.001)$.

S100A13 protein expression correlated positively with the intensity of VEGF-A staining (S100A13 $\leqslant 75 \%$, VEGF-A $0.6 \pm 1.0$ vs S100A13 > 75\%, VEGF-A $1.1 \pm 1.1, P=0.044$ ), but not with FGF-1. Indeed, only $26 \%$ of samples displaying $\leq 75 \%$ S100A13positive cells stained with VEGF antibodies, whereas the percentage of VEGF-A expressing tumors 


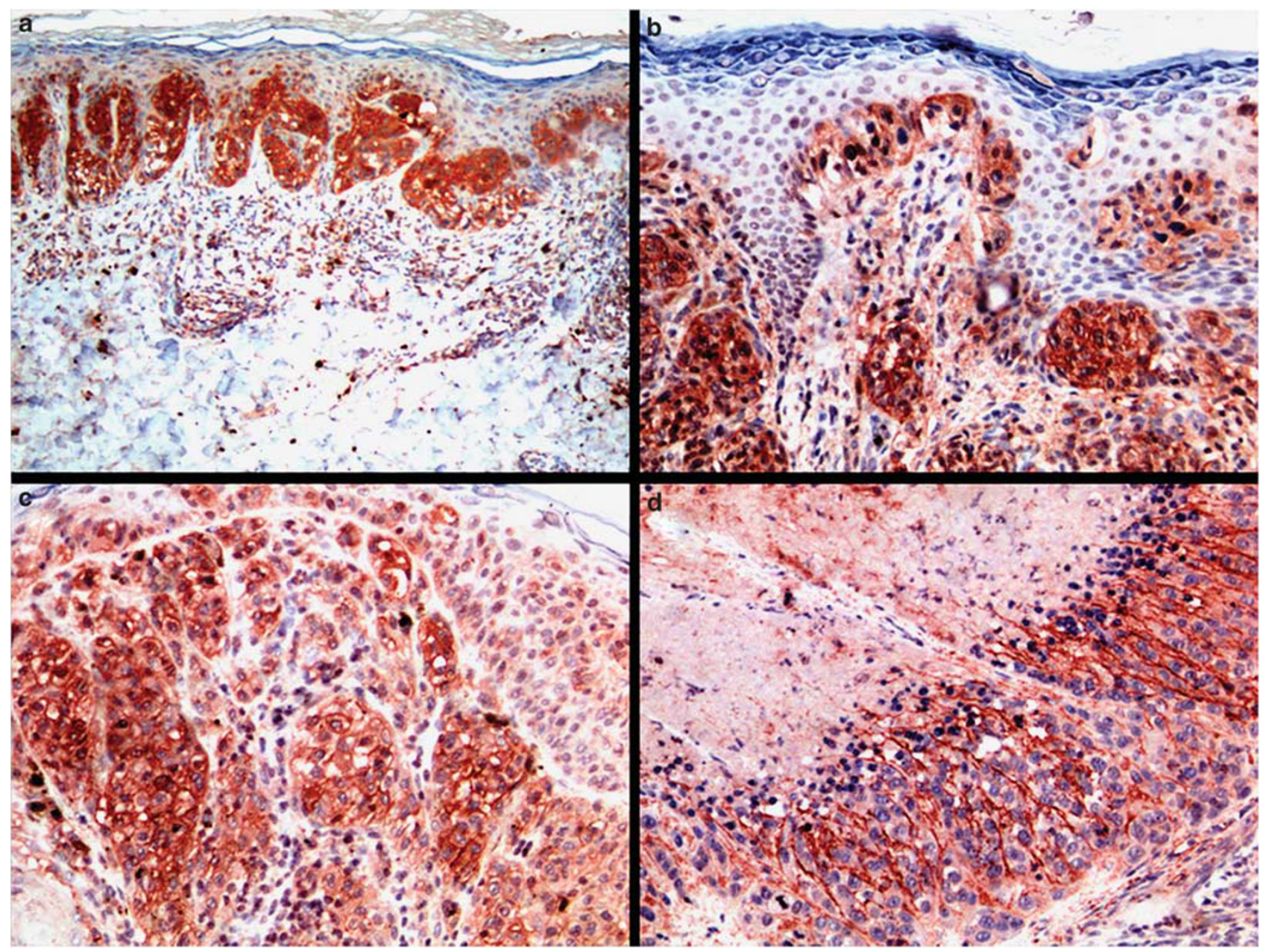

Figure 2 S100A13 staining in melanomas: (a) in situ melanoma with regression showing strong S100A13 cytoplasmic staining in neoplastic cells; (b) radial growth phase melanoma displays strong S100A13 immunostaining; single pagetoid melanoma cells in upward migration are also strongly positive; (c) strong cytoplasmic and membranous staining in vertical growth phase melanoma; (d) melanoma metastases with massive necrosis (upper left corner) shows moderate S100A13 positivity.

increased up to $56 \%$ when S100A13 was detectable in $>75 \%$ of cancer cells $(P=0.02)$. Moreover, S100A13 protein correlated positively with intratumoral microvessel density $(\beta=0.03 \pm 0.01 ; R=0.43$; $P=0.007)$.

Because of the smaller amount of samples analyzed by real-time PCR for S100A13 gene expression, mRNA levels correlated only with the intensity of VEGF-A staining $(P=0.023)$, but not with microvessel density.

\section{Expression of S100A13 Correlates with Outcome}

When the expression of S100A13 was analyzed in relation to several clinical parameters of outcome, we found that mRNA levels significantly correlated with TNM stage, risk of relapse and patient's status at follow-up (Figure 6). VEGF-A protein expression also correlated with TNM stage $(P=0.021)$, risk of relapse $(P=0.009)$ and status at follow-up $(P=0.004)$.

\section{Discussion}

Melanoma remains one of the most aggressive malignancies with poor survival rates for advanced metastatic disease. Tumor thickness has emerged as the main prognostic factor, with several additional tissue biomarkers, such as ulceration and mitotic activity, contributing to the prognostic stratification of patients. ${ }^{18-21}$

Although the ability of cutaneous melanoma cells to induce the formation of new blood vessels (angiogenesis) has been known for decades, ${ }^{22}$ the prognostic value of tumor vascularity remains somehow controversial. However, Neitzel et $a l^{23}$ found that the number of microvessels detected in primary lesions of metastatic melanomas was higher than those without metastases. Moreover, a recently published prospective study of 417 patients with cutaneous melanoma showed that tumor vascularity was the most important determinant of overall survival, performing better than tumor thickness. ${ }^{24}$ 


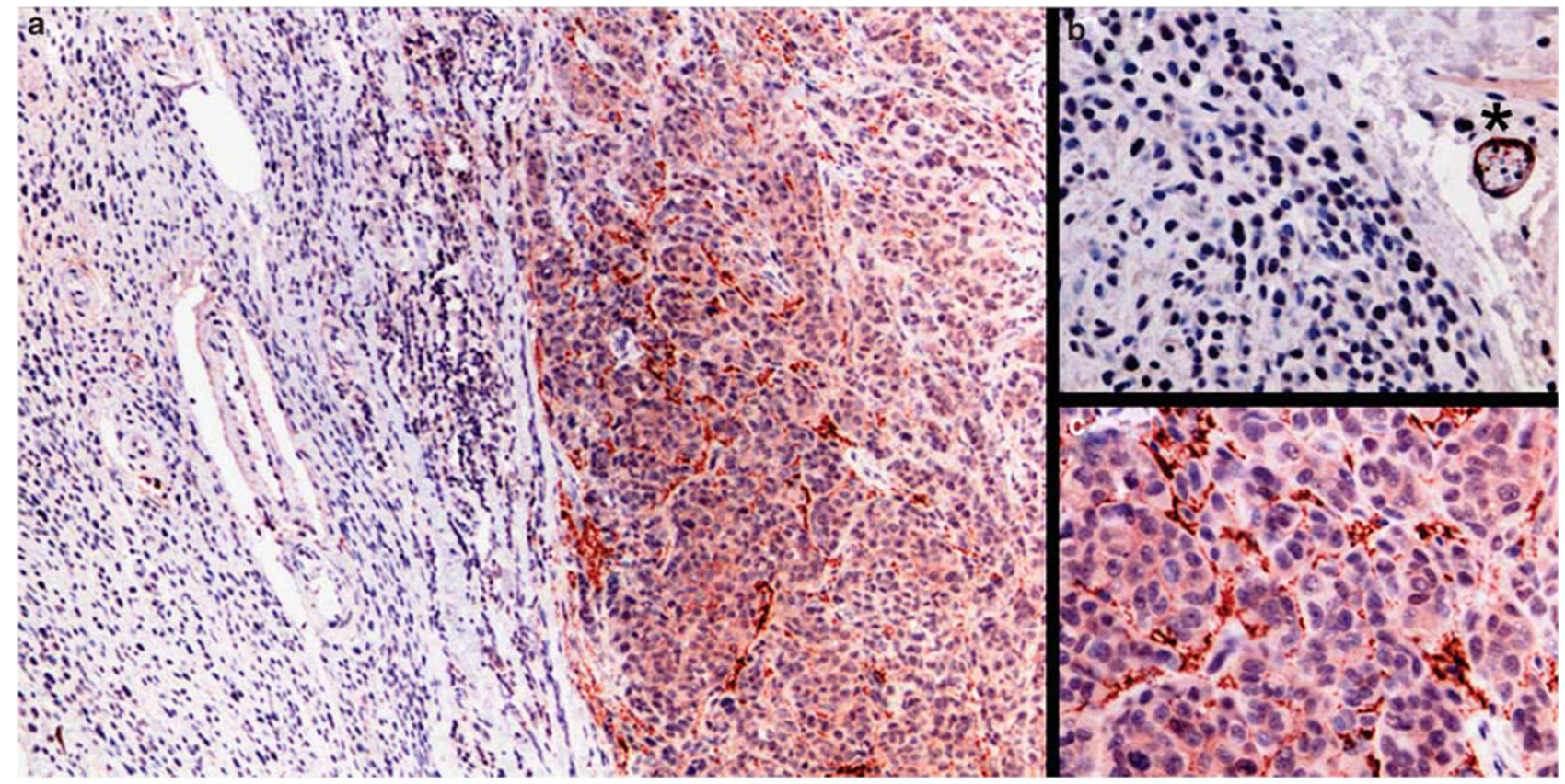

Figure 3 S100A13 staining in nevus-associated melanoma: (a) at low power magnification, nevus-associated melanoma shows cytologically bland melanocytes (left side) in contiguity with larger, pleomorphic atypical melanoma cells (right side); (b) higher magnification of the nevus component shows S100A13-negative melanocytes with an internal positive control (S100A13-positive nerve, asterisk); (c) higher magnification shows moderate S100A13 staining in the cytoplasm of melanoma cells.

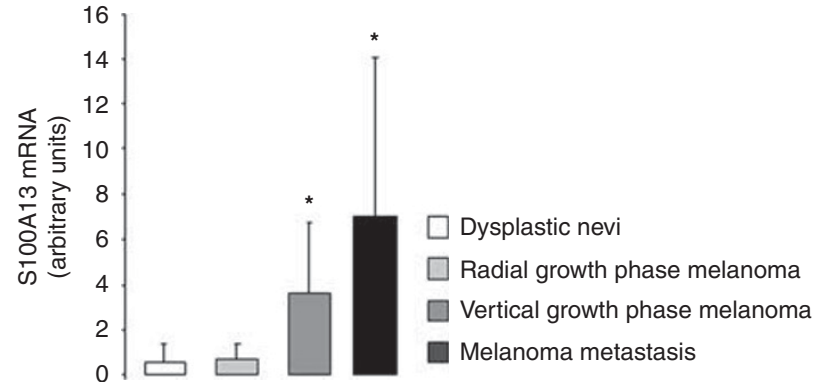

Figure 4 S100A13 mRNA levels in 'dysplastic' nevi, radial growth phase melanomas, vertical growth phase melanomas, melanoma metastases.

VEGF-A and the prototypes of the FGF family, FGF-1 and FGF-2, ${ }^{25}$ are the major angiogenic molecules produced by cutaneous melanomas. ${ }^{26-28}$ Several reports have associated VEGF-A with the angiogenic switch that initiates the vertical growth phase of malignant melanocytic lesions. ${ }^{4,29,30}$ Indeed, strong upregulation of VEGF-A has been correlated with progression of disease. ${ }^{31}$

During tumor angiogenesis, a well described cross-talk between VEGF-A and FGFs assures the correct formation of new blood vessels. ${ }^{1}$ Indeed, expression of dominant-negative forms of FGF receptor (FGFR)-1 and FGFR-2 triggers a downregulation of VEGF-A and decreases tumor vascularization. $^{32}$ Moreover, endothelial cells stimulated with FGF-2 upregulate the expression of FGFRs, VEGF-A and VEGF receptors. ${ }^{33,34}$ Thus, the full activation of the angiogenic phenotype in human malignancies seems to rely on the cooperation between VEGF-A and FGFs.

In our series, intratumoral microvessel density determined by CD105-a marker of angiogenic endothelial cells ${ }^{35}$-was found to be significantly higher in primary melanomas and metastases in comparison with common nevi, with melanomas in vertical growth being significantly more vascularized than tumors in radial growth. VEGF-A staining correlated with intratumoral and peritumoral vessel count and with tumor thickness, supporting the hypothesis that VEGF-A is a major regulator of the angiogenic switch in human melanomas. ${ }^{4,29,30}$

Because S100A13 is involved in the stressinduced secretion of FGF-1, ${ }^{11}$ we sought to determine whether this protein is expressed in melanocytic lesions and has a role in melanoma progression. To the best of our knowledge, this is the first time that S00A13 protein expression was evaluated in human melanocytic lesions. What we found was that S100A13 is expressed in 'dysplastic' nevi and in primary and metastatic melanomas. The expression of S100A13 was increasingly higher in more aggressive/advanced tumors (vertical growth phase and metastases compared with radial growth phase melanomas) and associated with invasion (Breslow thickness and Clark's level). Moreover, S100A13 correlated positively with the angiogenic indicators, VEGF-A and microvessel count.

S100A13 is a calcium- and copper-binding protein, belonging to the S100 family of polypeptides. ${ }^{7}$ Under stress conditions (heat shock, hypoxia, 


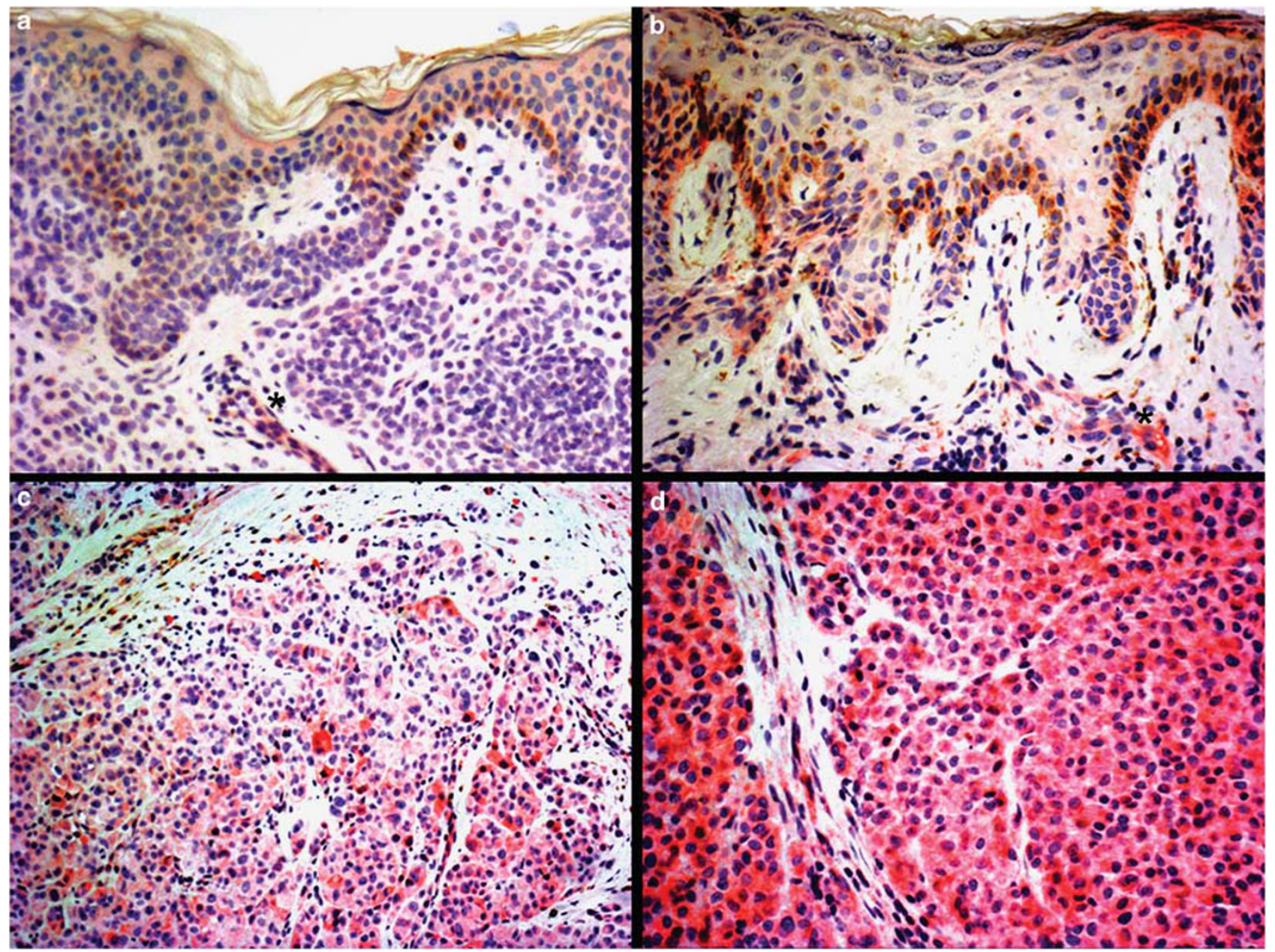

Figure 5 VEGF-A staining in melanocytic nevi and melanomas: (a) no staining is observed in melanocytes of a common acquired melanocytic nevus, whereas endothelial cells decorating a capillary vessel inside the lesion show weak VEGF-A positivity (asterisk); (b) 'dysplastic' nevus showing mild VEGF-A staining in $25-50 \%$ of melanocytes (score 1); endothelial cells of a capillary vessel show moderate positivity (asterisk); (c) vertical growth phase melanoma displays moderate immunostaining in 50-75\% of cells (score 2); (d) strong VEGF-A staining in more than $75 \%$ of cells in melanoma metastases (score 3 ).

starvation) and in the presence of copper, S100A13 mediates the assemble of the multiprotein complex, comprising S100A13, FGF-1, the extravesicular portion of synaptotagmin-1 (p40-syt 1) and sphingosine kinase 1 , that facilitates the release of FGF-1. ${ }^{10}$ S100A13 is necessary for FGF-1 secretion, as shown by the ability of a dominant negative form of the protein to inhibit the release of the growth factor when co-transfected with FGF-1 into NIH 3T3 cells. ${ }^{11}$ Moreover, it has been demonstrated that secreted FGF-1 is a homodimer obtained through the oxidation of cysteine residues, and that dimerization is necessary for the release of the growth factor, as a cys-free FGF-1 mutant cannot be exported, in presence of physiological levels of S100A13. ${ }^{36} \mathrm{By}$ contrast, when S100A13 protein expression is forced in NIH 3T3 cells, the FGF-1 cys-free mutant is released into the extracellular compartment overcoming the requirement for dimerization. ${ }^{11}$

A recent report indicated that upregulation of S100A13 and VEGF-A correlates with activation of angiogenesis in high-grade human astrocytic gliomas. ${ }^{8}$ Moreover, an interesting report has described the overexpression of S100A13 and FGF-1 in endometriosis, suggesting a possible role for this protein in the process of endometriotic angiogenesis. ${ }^{37,38}$

In our study, FGF-1 was expressed in all tissues examined, without any difference between benign and malignant lesions. We propose that the upregulation of S100A13, in an FGF-1-rich background, may force the export of FGF-1 from the cytoplasm to the extracellular space, where the growth factor may interact with its target cells and cooperate with VEGF-A in promoting the angiogenic switch that favors the progression of melanoma lesions. Indeed, using A375 cells, a model of human melanoma, we previously demonstrated that the release of endogenous FGF-1, under conditions of stress, is inhibited by the copper chelator ammonium tetrathiomolibdate, suggesting that the S100A13dependent, copper-mediated secretory complex is 


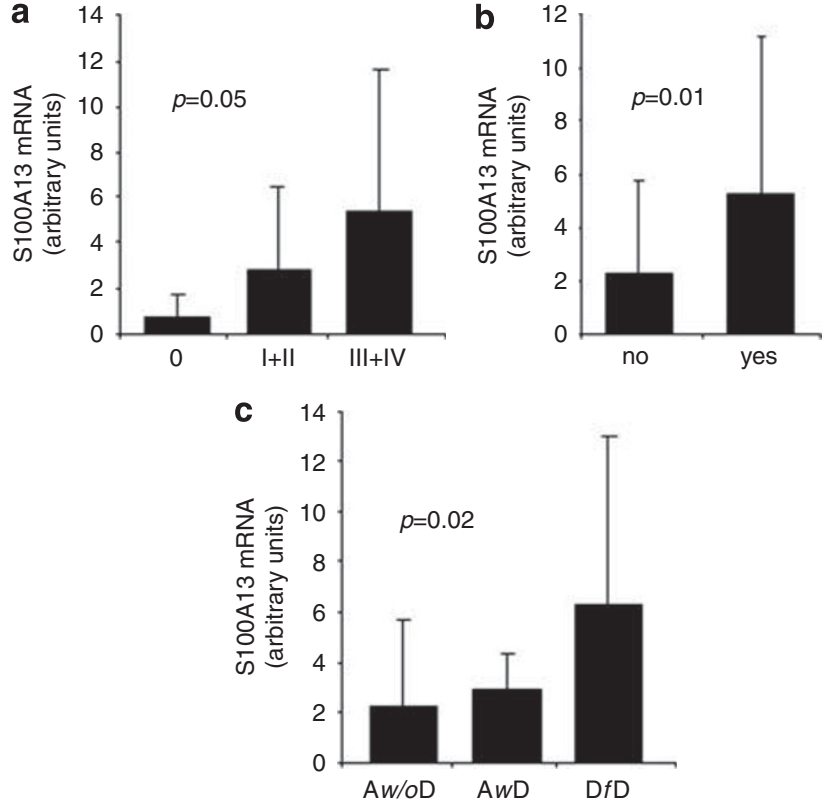

Figure 6 Correlation between S100A13 mRNA levels and stage (a), progression of disease (b) and status at follow-up (c). Aw/oD, alive without disease, AwD alive with disease, DfD, dead for disease.

required for the export of FGF-1 from melanoma cells, in vitro. ${ }^{39}$

Several members of the S100 family are considered tumor markers. ${ }^{40}$ In melanoma, the level of secreted S100B reflects tumor load and correlates with stage and prognosis, still remaining the most sensitive immunohistochemical marker of the disease $^{12}$ and an independent prognostic factor. ${ }^{13}$ Melanoma cells also express S100A2, S100A4 and S100A6. S100B and S100A4 inhibit p53 phosphorylation, compromising its tumor-suppressor activity. ${ }^{16}$ Moreover, S100A4 supports numerous cellular activities that may favor metastatization, including cell motility, invasion, apoptosis and angiogenesis. ${ }^{41}$ Elevated S100A6 gene expression was found in highly metastatic melanomas compared with low metastatic lesions, benign nevi and normal skin, indicating this protein as a progression marker. ${ }^{42}$ In contrast, S100A2 seems to exert a tumor suppressor activity, most likely through inhibition of Cox2 which is associated with tumorigenesis in many cancers, and its expression is reduced in melanoma. ${ }^{14}$

In lung cancer-derived cell lines, transcriptional analysis of gene expression identified S100A13 in more aggressive, invasive phenotypes. Depletion of S100A13 by gene silencing, resulted in a significant decrease of their invasive potential, in vitro. ${ }^{9}$ Moreover, S100A13 was detected in tumor cells circulating in blood of patients with metastatic cancer, indicating that this protein may be considered a predictor of metastases. ${ }^{43}$ We have no data to propose an association between S100A13 and the metastatic potential of melanoma cells; however, it will be of interest to further explore this issue.

In conclusion, although tumor thickness is considered the main prognostic factor in melanoma, numerous additional histological and molecular markers have emerged over time for their ability to better define the risk profile of a single patient and to guide treatment decisions. We propose S100A13 as an indicator of the angiogenic switch that facilitates disease progression and as a promising prognostic marker, based on the positive correlation identified between S100A13 mRNA levels, risk of relapse and status of patients at follow-up. However, additional studies on larger series will be necessary to establish whether expression of S100A13 is an independent prognostic factor in patients with melanoma.

\section{Acknowledgements}

This study was supported by grant from Fondazione Ente Cassa di Risparmio di Pistoia e Pescia (20082009) to DM and FT; Grant 26/08 from Lega Italiana per la Lotta contro i Tumori and 2009 Grant from Fondazione Berlucchi and from AIRC to ML; NIH RO1 HL035627 grant and Maine Cancer Foundation grant to IP.

\section{Disclosure/conflict of interest}

The authors declare no conflict of interest.

\section{References}

1 Presta M, Dell'Era P, Mitola S, et al. Fibroblast growth factor/fibroblast growth factor receptor system in angiogenesis. Cytokine Growth Factor Rev 2005;16: 159-178.

2 Gerwins P, Skoldenberg E, Claesson-Welsh L. Function of fibroblast growth factors and vascular endothelial growth factors and their receptors in angiogenesis. Crit Rev Oncol Hematol 2000;34:185-194.

3 Straume O, Akslen LA. Expression of vascular endothelial growth factor, its receptors (FLT-1, KDR) and TSP-1 related to microvessel density and patient outcome in vertical growth phase melanomas. Am J Pathol 2001;159:223-235.

4 Bayer-Garner IB, Hough Jr AJ, Smoller BR. Vascular endothelial growth factor expression in malignant melanoma: prognostic versus diagnostic usefulness. Mod Pathol 1999;12:770-774.

5 Mahabeleshwar GH, Byzova TV. Angiogenesis in Melanoma. Semin Oncol 2007;34:555-565.

6 Marenholz I, Heinzman CW, Fritz G. S100 protein in mouse and man: from evolution to function and pathology. Biochem Biophys Res Commun 2004;322: 1111-1122.

7 Santamaria-Kisiel L, Rintala-Dempsey AC, Shaw GS. Calcium dependent and independent interactions of the S100 protein family. Biochem J 2006;396:201-214. 
8 Landriscina M, Schinzari G, Di Leonardo G, et al. S100A13, a new marker of angiogenesis in human astrocytic gliomas. J Neurooncol 2006;80:251-259.

9 Pierce A, Barron N, Linehan R, et al. Identification of a novel, functional role for S100A13 in invasive lung cancer cell lines. Eur J Cancer 2008;44:151-159.

10 Prudovsky I, Tarantini F, Landriscina $\mathrm{M}$, et al. Secretion without Golgi. J Cell Biochem 2008;103: 1327-1343.

11 Landriscina M, Soldi R, Bagalá C, et al. S100A13 participates in the release of fibroblast growth factor 1 in response to heat shock in vitro. J Biol Chem 2001;276:22544-22552.

12 Ohsie SJ, Sarantopoulos GP, Cochran AJ, et al. Immunohistochemical characteristics of melanoma. J Cutan Pathol 2008;35:433-444.

13 von Schoultz E, Hansson LO, Djureen E, et al. Prognostic value of serum analyses of S100B protein in malignant melanoma. Melanoma Res 1996;6: 133-137.

14 Maelandsmo G, Florenes VA, Mellingsaeter T, et al. Differential expression patterns of S100A2, S100A4 and S100A6 during progression of human malignant melanoma. Int J Cancer 1997;74:464-469.

15 Andersen K, Nesland JM, Holm R, et al. Expression of S100A4 combined with reduced E-cadherin expression predicts patient outcome in malignant melanoma. Modern Pathol 2004;17:990-997.

16 Salama I, Malone PS, Mihaimeed F, et al. A review of the S100 proteins in cancer. Eur J Surg Oncol 2008;34: 357-364.

17 Massi D, De Nisi MC, Franchi A, et al. Inducible nitric oxide synthase expression in melanoma: implications in lymphangiogenesis. Modern Pathol 2009;22:21-30.

18 Kashani-Sabet M, Leong SPL, Sagebiel RW. Prognostic factors in malignant melanoma. Surg Oncol Clin N Am 1997;6:599-623.

19 Balch CM, Buzaid AC, Soong SJ, et al. Final version of the American Joint Committee on Cancer staging system for cutaneous melanoma. J Clin Oncol 2001;19:3635-3648.

20 Gogas H, Eggermont AM, Hauschild A, et al. Biomarkers in melanoma. Ann Oncol 2009;6:vi8-v13.

21 Balch CM, Gershenwald JE, Soong SJ, et al. Final Version of 2009 AJCC Melanoma Staging and Classification. J Clin Oncol 2009;27:6199-6206.

22 Reed RJ, Ichinose H, Clark Jr WH. et al. Common and uncommon melanocytic nevi and borderline melanomas. Semin Oncol 1975;2:105-118.

23 Neitzel LT, Neitzel CD, Magee KL, et al. Angiogenesis correlates with metastases in melanoma. Ann Surg Oncol 1999;6:70-74.

24 Kashani-Sabet M, Sagebiel RW, Ferreira CM, et al. Tumor vascularity in the prognostic assessment of primary cutaneous melanoma. J Clin Oncol 2002;20:1826-1831.

25 Maciag T, Mehlman T, Friesel R, et al. Heparin binds endothelial cell growth factor, the principal endothelial cell mitogen in bovine brain. Science 1984;225: 932-935.

26 Reed JA, McNutt NS, Albino AP. Differential expression of basic fibroblast growth factor (bFGF) in melanocytic lesions demonstrated by in situ hybridization. Implications for tumor progression. Am J Pathol 1994;144:329-336.
27 Detmar M. Molecular regulation of angiogenesis in the skin. J Invest Dermatol 1996;106:207-208.

28 Xerri L, Battyani Z, Grob J-J, et al. Expression of FGF1 and FGFR1 in human melanoma tissues. Melanoma Res 1996;6:223-230.

29 Barnhill RL, Levy MA. Regressing thin cutaneous malignant melanomas $(<$ or $=1.0 \mathrm{~mm}$ ) are associated with angiogenesis. Am J Pathol 1993;143:99-104.

30 Marcoval J, Moreno A, Graells J, et al. Angiogenesis and malignant melanoma. Angiogenesis is related to the development of vertical (tumorigenic) growth phase. J Cutan Pathol 1997;24:212-218.

31 Brychtova S, Bezdekova M, Brychta T, et al. The role of vascular endothelial growth factors and their receptors in malignant melanomas. Neoplasma 2008;55: 273-279.

32 Auguste P, Gürsel DB, Lemière S, et al. Inhibition of fibroblast growth factor/fibroblast growth factor receptor activity in glioma cells impedes tumor growth by both angiogenesis-dependent and -independent mechanisms. Cancer Res 2001;61:1717-1726.

33 Seghezzi G, Patel S, Ren CJ, et al. Fibroblast growth factor-2 (FGF-2) induces vascular endothelial growth factor (VEGF) expression in the endothelial cells of forming capillaries: an autocrine mechanism contributing to angiogenesis. J Cell Biol 1998;141: 1659-1673.

34 Gabler C, Plath-Gabler A, Killian GK, et al. Expression pattern of fibroblast growth factor (FGF) and vascular endothelial growth factor (VEGF) system members in bovine corpus luteum endothelial cells during treatment with FGF-2, VEGF or oestradio. Reprod Domest Anim 2004;39:321-327.

35 Duff SE, Li C, Garland JM, et al. CD105 is important for angiogenesis: evidence and potential applications. FASEB J 2003;17:984-992.

36 Tarantini F, Gamble S, Jackson A, et al. The cysteine residue responsible for the release of fibroblast growth factor-1 residues in a domain independent of the domain for phosphatidylserine binding. J Biol Chem 1995;270:29039-29042.

37 Hayrabedyan S, Kyurkchiev S, Kehayov I. FGF-1 and S100A13 possibly contribute to angiogenesis in endometriosis. J Reprod Immunol 2005;67:87-101.

38 Hayrabedyan S, Kyurkchiev S, Kehayov1 I. Endoglin (cd105) and S100A13 as markers of active angiogenesis in endometriosis. Reprod Biol 2005;5:51-67.

39 Di Serio C, Doria L, Pellerito S, et al. The release of fibroblast growth factor-1 from melanoma cells requires copper ions and is mediated by phosphatidylinositol 3-kinase/Akt intracellular signaling pathway. Cancer Lett 2008;267:67-74.

40 Sedaghat F, Notopoulos A. S100 protein family and its application in clinical practice. Hippokratia 2008;4: 198-204.

41 Helfman DM, Kim EJ, Lukanidin E, et al. The metastases associated protein S100A4: role in tumour progression and metastases. Br J Cancer 2005;92: 1955-1958.

42 Weterman MA, Van Mujen GN, Bloemers HP, et al. Expression of calcyclin in human melanocytic lesions. Cancer Res 1993;53:6061-6066.

43 Smirnov DA, Zweitzig DR, Foulk BW, et al. Global gene expression profiling of circulating tumor cells. Cancer Res 2005;65:4993-4997. 